\title{
The Associations of Coping Mechanism with Arterial Stiffness in Hwa-Byung Patients
}

\author{
Yu Jin Lee ${ }^{1}$ \\ Kyung Won Baek ${ }^{2}$ \\ Kyu Wol Yun ${ }^{3}$ \\ Wonshin Lim ${ }^{4}$ \\ Weonjeong $\mathrm{Lim}^{3}$ \\ ${ }^{1}$ Department of Psychiatry, \\ Gachon University \\ of Medicine and Science, \\ Gil Hospital, Incheon, \\ 2Department of Psychiatry, \\ College of Medicine, \\ Hanyang University, Seoul, \\ 3Department of Psychiatry, \\ College of Medicine, \\ Ewha Womans University, \\ Seoul, \\ 4Department of Child Welfare, \\ Namseoul University, \\ Cheonan, Korea
}

\section{Correspondence \\ Weonjeong Lim, MD, PhD Department of Psychiatry, College of Medicine, Ewha Womans University, 911 Mok-dong, Yangcheon-gu, Seoul 158-710, Korea \\ Tel +82-2-2650-5164 \\ Fax +82-2-2650-5164 \\ E-mail psyweon@ewha.ac.kr}

(c) This is an Open Access article distributed under the terms of the Creative Commons Attribution Non-Commercial License (http://creativecommons.org/licenses/by-nc/3.0) which permits unrestricted non-commercial use, distribution, and reproduction in any medium, provided the original work is properly cited.
Objective The goal of this study is to assess the relationship between stress coping mechanisms and the risk of atherosclerosis in patients with Hwa-Byung.

Methods The Korean version of the Ways of Coping Checklist (WOCC) was administered to 50 patients with Hwa-Byung (49.1 \pm 10.1 years, 6 males). Brachial-ankle pulse wave velocity (baPWV) and serum cholesterol level were assessed in all participants.

Results After controlling for age, sex, diagnosis of hypertension, Body Mass Index (BMI), and serum cholesterol level, the score of seeking social support in coping strategies was negatively correlated with right and left baPWV $(r=-0.356, p=0.016 ; r=-0.373, p=0.012$, respectively). In addition, the score of active coping mechanism was negatively correlated with both sides of baPWV ( $r=-0.383, p=0.009 ; \mathrm{r}=-0.389, \mathrm{p}=0.008$, respectively).

Conclusion The seeking social support and active coping mechanism were inversely related to the severity of arterial stiffness in Hwa-Byung patients. Therefore, our result may suggest a possibility that coping strategies in Hwa-Byung patients are associated with the risk of atherosclerosis.

Psychiatry Invest 2009;6:241-244

Key Words Hwa-Byung, Coping mechanism, Atherosclerosis.

Received: June 17, 2009 Revised: July 29, 2009 Accepted: August 15, 2009 Available online: November 5, 2009

\section{Introduction}

Psychological stress is an inevitable element of daily living. The role of psychological stress on health issues depends on subjective appraisals, types of coping strategies or availability of social supports in individuals. ${ }^{1,2}$ Mental stress is known to be associated with cardiovascular diseases including atherosclerosis and coronary artery disease in prior studies. ${ }^{3-5}$ It has also a prolonged unfavourable effect on arterial stiffness and wave reflections via regulators such as catecholamine and endothelial function. ${ }^{6-8}$ Increased arterial stiffness is one of the pathological states of vascular diseases, and is closely associated with atherosclerotic cardiovascular disease. In prior study, the associations between job stress and arterial stiffness were shown as well. ${ }^{9}$ Therefore, how to deal with stress, so called coping mechanism, could play an important role in the risk of atherosclerosis. ${ }^{10}$ In addition, individuals with maladaptive coping strategies were suggested to be more vulnerable to psychiatric difficulties including depression and anxiety, which could be cardiovascular risk factors. ${ }^{11}$

Pulse wave velocity (PWV) is known to be a useful and non-invasive indicator of arterial stiffness, ${ }^{12}$ and the measurement of brachial-ankle PWV (baPWV) has recently been applied as a reliable marker for atherosclerosis in general population. ${ }^{13}$ The time delay between the rapid upstroke of pulse waves between the brachial artery and the ankle artery simultaneously is recorded. PWV was defined as the ratio between the distance and the time delay. ${ }^{14}$

Hwa-Byung is a well-known Korean culture bound syndrome with high preval- 
ence among middle-aged married women of lower socioeconomic status and lower educational background. ${ }^{15,16}$ Patients with Hwa-Byung have various psychological and somatic symptoms including depressed mood, anxiety, pushing-up in the chest, chest tightness, and so on. ${ }^{15,16}$ In terms of the relationship between Hwa-Byung and cardiovascular disease, their somatic complaints often could be confused with the symptoms of cardiovascular disease. In addition, characteristics of Hwa-Byung such as the age of prevalence, low socioeconomic status and psychiatric symptoms could be related to various risk factors of cardiovascular disease. ${ }^{17}$

Prior studies reported that Hwa-Byung patients frequently used ineffective coping strategies such as active forgetting, accommodation, fatalism, emotional pacification and emotional support seeking. ${ }^{18}$ In addition, they frequently employed passive withdrawal, self-criticism, perseverance, seeking of social support and religious guidance in stressful situations. ${ }^{16}$

In this study, we hypothesized that characteristics of stress coping mechanisms would be related to arterial stiffness in Hwa-Byung patients. We aimed at investigating the association of coping strategies with the risk of atherosclerosis assessed by baPWV in individuals with Hwa-Byung.

\section{Methods}

Initially, fifty-five patients who visited Hwa-Byung clinic participated in current study. Hwa-Byung was diagnosed based on the participants' responses to two questions. ${ }^{19}$ 1) Do you think you have Hwa-Byung? 2) Do others think you have Hwa-Byung? Finally, 50 patients (49.1 \pm 10.1 years, 6 males) who answered "yes" to both questions were enrolled. Gathering of demographic information, medical history and the Korean version of the Ways of Coping Checklist (WOCC) ${ }^{20}$ were administered to final subjects. None had history of serious medical disease.

WOCC was developed by Folkman and Lazarus ${ }^{21}$ to assess how to deal with stress. The Korean version of this scale was validated by Kim and Lee. ${ }^{20}$ It consists of 62 items measuring each stress coping strategy of problem-focused (analyse the problem or plan to act), seeking social support (talk to someone and get support and advice), emotional-focused (attempts to regulate emotional states that result from the stressful situation) and wishful thinking (wish or hope). In this checklist, coping mechanisms of problem-focused and seeking social support are classified into the active coping, and emotionalfocused and wishful thinking into the passive coping. ${ }^{20,21}$

On the day for measuring baPWV and serum choleste- rol level, all subjects were instructed to refrain from breakfast, coffee, and smoking on the morning of check-up. BaPWV were measured using automatic waveform analyzer (VP-1000 type 230, Japan). Measurements were taken with subjects lying in a supine position after resting at least 5 min. PWV (in centimetres per second) was calculated as the ratio between distance traveled by the pulse wave and the time delay. The average of at least 10 successive measurements was used in the analysis. An increase of baPWV indicates increased arterial stiffening.

After controlling for age, sex, diagnosis of hypertension, Body Mass Index (BMI), and serum cholesterol level which are known as risk factors of atherosclerosis, ${ }^{22}$ partial correlation analysis between the score of each strategy in the coping checklist and baPWV was performed by Statistical Package for Social Science (SPSS; SPSS Inc, Chicago, IL, USA) 11.5 program. The statistical significance level in current study was set at $\mathrm{p}<0.05$.

\section{Results}

Table 1 showed the mean scores of WOCC subscales and of baPWV, as well as the demographic characteristics of subjects. After controlling for age, sex, diagnosis of hypertension, BMI, and serum cholesterol level, the score of seeking social support in WOCC was negatively correlated with right and left baPWV ( $\mathrm{r}=-0.356$, $\mathrm{p}=0.016 ; \mathrm{r}=-0.373, \mathrm{p}=0.012$, respectively). In addition, the score of active coping mechanism, i.e., the sum score

TABLE 1. Demographic characteristics, scores of WOCC and baPWV $(\mathrm{N}=50)$

\begin{tabular}{lc}
\hline & Mean \pm SD \\
\hline Age (years) & $49.1 \pm 10.1$ \\
Sex & $44(88.0 \%)$ \\
Female & $6(12.0 \%)$ \\
Male & \\
Active coping & $29.48 \pm 9.90$ \\
$\quad$ Problem-focused & $16.42 \pm 7.21$ \\
Seeking social support & $46.14 \pm 10.55$ \\
Total & \\
Passive coping & $29.56 \pm 9.75$ \\
Emotion-focused & $23.44 \pm 7.13$ \\
Wishful thinking & $52.22 \pm 11.69$ \\
Total & \\
BaPWV (cm/sec) & $1368.20 \pm 231.31$ \\
Right & $1373.58 \pm 225.86$ \\
Left & $23.21 \pm 2.53$ \\
Body Mass Index & $12(24 \%)$ \\
Dx of hypertension & $190.60 \pm 38.01$ \\
Serum cholesterol (mg\%) & \\
\hline WOCC: the Ways of Coping Checklist, baPWV: brachial-ankle \\
pulse wave velocity
\end{tabular}


TABLE 2. Partial correlations between score of WOCC and $\operatorname{baPWV}(\mathrm{N}=50)$

\begin{tabular}{lcc}
\hline & BaPWV (right) & BaPWV (left) \\
\hline Active coping & $-0.38^{* *}$ & $-0.39^{* *}$ \\
Problem-focused & -0.14 & -0.14 \\
Seeking social support & $-0.36^{*}$ & $-0.37^{*}$ \\
Passive coping & 0.14 & 0.17 \\
Emotion-focused & 0.15 & 0.16 \\
Wishful thinking & 0.02 & 0.03 \\
\hline
\end{tabular}

All values calculated after controlling for age, sex, diagnosis of hypertension, Body Mass Index, and serum cholesterol level. ${ }^{*} p<0.05,{ }^{*} p<0.01$. WOCC: the Ways of Coping Checklist, baPWV: brachial-ankle pulse wave velocity

of problem-focused coping and seeking social support, was negatively correlated with both sides of baPWV $(r=$ $-0.383, p=0.009 ; r=-0.389, p=0.008$, respectively). Scores of other subscales in WOCC had no significant correlation with baPWV (Table 2).

\section{Discussion}

In this study, we found the inverse relationship of the seeking social support to arterial stiffness assessed by baPWV in Hwa-Byung patients. In addition, active coping strategies including problem-focused and seeking social support were negatively correlated with baPWV.

The core concept of Hwa-Byung is thought to be originated from the emotion of "Hahn" which is defined as mixed feelings of sorrow and anger in Korean culture. ${ }^{23}$ It is known to be caused by long-standing psychosocial stress of familial discords, economic difficulties, troubles with mother-in-law or sister-in-laws and unfair treatments by in-laws. ${ }^{24}$ And, managing suppressed or repressed anger is the most important dynamic mechanism in the concept of Hwa-Byung. ${ }^{23}$ Therefore, the pathogenesis of Hwa-Byung could be related to maladaptive coping to chronic stress. Hwa-Byung patients have been suggested to deal with their stress by more passive or emotional coping mechanisms. ${ }^{16,18}$

Regarding psychiatric symptoms in Hwa-Byung, prior studies suggested that symptoms of Hwa-Byung may be closely related to those in somatization disorder, generalized anxiety disorder, depression or panic disorder among diagnostic categories of the western medicine. ${ }^{25}$

Maladaptive coping mechanisms could worsen psychiatric symptoms of depression, somatization and anxiety. ${ }^{11}$ Therefore, adaptive and/or maladaptive coping mechanism might be meaningful in physical and mental health of individuals with Hwa-Byung.

Depressed mood, anxiety and psychosocial stress have been known to be aggravating factors of mortality in cardiovascular diseases. ${ }^{26,27}$ Yeragani et al. ${ }^{28}$ noted in- creased PWV in patients were associated with anxiety. Furthermore, carotid-femoral PWV was suggested to have association with depression. ${ }^{14}$

In this study, less use of seeking social support coping was related to increased risk for atherosclerosis in HwaByung patients. This result is supported by previous notion of which social deprivation is one of predictors on atherosclerotic progression in general population. ${ }^{29} \mathrm{An}-$ other plausible explanation might be that more severe depression and/or anxiety as risk factors of atherosclerosis in Hwa-Byung could also play negative roles on their social relationships from which they can get support. In addition, we found that the active coping mechanism was inversely associated with the arterial stiffness of Hwa-Byung patients. This association could be mainly caused by its relationship with the coping strategy of seeking social support. However, the significance level in the association with active coping mechanism was higher than that in relation to only the seeking social support. There was a tendency of negative correlation of problem focused solving with the severity of arterial stiffness, although it was not statistically significant. Problem focused solving involves strategies that reduce stress by determining the best solution and means to achieve. When individuals believe that nothing can be done to deal with the stress, they employ emotional coping mechanisms including wishful thinking or avoidance. ${ }^{30}$ Problem focuses process might be performed under healthier ego function and/or less stressful event. ${ }^{30}$ Ineffective coping with less active strategies in Hwa-Byung patients was related to increased risk of atherosclerosis in this study.

No significant relationship between passive coping strategies and baPWV was found in current study. Prior notion, which individuals with higher diastolic blood pressure employed more frequently passive coping strategies of wishful thinking, avoidance, and minimization of threat, was inconsistent with current result. ${ }^{31}$ This discrepancy may be caused by different characteristics of study subjects of general employees from current study. Coping strategies in Hwa-Byung patients might be quite different and more maladaptive from those of general population. ${ }^{16,18}$ Further research with larger sample size will elucidate this issue more clearly.

There were several limitations in current study. Small sample size, no comparison group, no information of smoking status, and no objective assessment of psychiatric symptoms might limit generalizations of current results.

However, to the best of our knowledge, this is the first study which investigated the association of coping style with the risk of atherosclerosis in individuals with HwaByung. Moreover, it can be one of strengths of our study 
to control statistically well-known contributing factors of atherosclerosis such as age, sex, diagnosis of hypertension, BMI, and serum cholesterol level.

In summary, we reported that coping styles to stress was related to arterial stiffness in Hwa-Byung patients. Therefore, our result may suggest a possibility that education and strengthening adaptive coping strategies in Hwa-Byung patients reduce the risk of atherosclerosis.

\section{REFERENCES}

1. Lazarus RS. A strategy for research on psychological and social factors in hypertension. J Human Stress 1978;4:35-40.

2. Wilder JF. Coping with the stress of change. Internist 1992;33:11-12.

3. Williams JK, Vita JA, Manuck SB, Selwyn AP, Kaplan JR. Psychosocial factors impair vascular responses of coronary arteries. Circulation 1991;84:2146-2153.

4. Manuck SB, Marsland AL, Kaplan JR, Williams JK. The pathogenicity of behavior and its neuroendocrine mediation: an example from coronary artery disease. Psychosom Med 1995;57:275-283.

5. Dimsdale JE, Hackett TP, Hutter AM Jr, Block PC, Catanzano D. Type A personality and extent of coronary atherosclerosis. Am J Cardiol 1978;42:583-586.

6. Wilkinson IB, Franklin SS, Cockcroft JR. Nitric oxide and the regulation of large artery stiffness: from physiology to pharmacology. Hypertension 2004;44:112-116.

7. Wilkinson IB, MacCallum H, Hupperetz PC, van Thoor CJ, Cockcroft JR, Webb DJ. Changes in the derived central pressure waveform and pulse pressure in response to angiotensin II and noradrenaline in man. J Physiol 2001;530:541-550.

8. Vlachopoulos C, Kosmopoulou F, Alexopoulos N, Ioakeimidis N, Siasos G, Stefanadis C. Acute mental stress has a prolonged unfavorable effect on arterial stiffness and wave reflections. Psychosom Med 2006;68:231-237.

9. Nomura K, Nakao M, Karita K, Nishikitani M, Yano E. Association between work-related psychological stress and arterial stiffness measured by brachial-ankle pulse-wave velocity in young Japanese males from an information service company. Scand J Work Environ Health 2005;31:352-359.

10. Bugajska J, Widerszal-Bazyl M, Radkiewicz P, Pasierski T, Szulczyk GA, Zabek J, et al. Perceived work-related stress and early atherosclerotic changes in healthy employees. Int Arch Occup Environ Health 2008;81:1037-1043.

11. Manos N, Christakis J. Coping with cancer: psychological dimensions. Acta Psychiatr Scand 1985;72:1-5.

12. Lehmann ED. Clinical value of aortic pulse-wave velocity measurement. Lancet 1999;354:528-529.

13. Yamashina A, Tomiyama H, Takeda K, Tsuda H, Arai T, Hirose K, et al. Validity, reproducibility, and clinical significance of noninvasive brachial-ankle pulse wave velocity measurement. Hypertens Res 2002;25:359-364

14. Tiemeier H, Breteler MM, Popele NM, Hofman A, Witteman JC. Latelife depression is associated with arterial stiffness: a population-based study. J Am Geriatr Soc 2003;51:1105-1110.

15. Min SK. A study of the concept of Hwabyung. J Korean Neuropsychiatr Asso 1989;28:604-616.

16. Park YJ, Kim HS, Kang HC, Kim JW. A survey of Hwa-Byung in middle-age Korean women. J Transcult Nurs 2001;12:115-122.

17. Williams RB, Barefoot JC, Califf RM, Haney TL, Saunders WB, Pryor DB, et al. Prognostic importance of social and economic resources among medically treated patients with angiographically documented coronary artery disease. JAMA 1992;267:520-524.

18. Chon KK, Kim JW, Park HK. Life stress and coping styles of HwaByung patients and healthy controls. Korean Journal of Stress Research 1998;6:9-23.

19. Lin KM, Lau JK, Yamamoto J, Zheng YP, Kim HS, Cho KH, et al. Hwa-byung. A community study of Korean Americans. J Nerv Ment Dis 1992;180:386-391.

20. Kim JH, Lee JH. The relationship between factor of stress coping mechanism and depression. Korean J Behav Sci 1985;7:127-138.

21. Folkman S, Lazarus RS. Coping as a mediator of emotion. J Pers Soc Psychol 1988;54:466-475.

22. Fogari R, Zoppi A. Heart and hypertension. Am J Hypertens 1989; 2:16S-23S.

23. Park YJ, Kim HS, Schwartz-Barcott D, Kim JW. The conceptual structure of hwa-byung in middle-aged Korean women. Health Care Women Int 2002;23:389-397.

24. Choi YJ, Lee KJ. Evidence-based nursing: effects of a structured nursing program for the health promotion of Korean women with Hwa-Byung. Arch Psychiatr Nurs 2007;21:12-16.

25. Min SK, Kim JH. A study on Hwa-Byung in Bogil Island. J Korean Neuropsychiatr Asso 1986;25:459-466.

26. Weissman MM, Markowitz JS, Ouellette R, Greenwald S, Kahn JP. Panic disorder and cardiovascular/cerebrovascular problems: results from a community survey. Am J Psychiatry 1990;147:1504-1508.

27. Carney RM, Freedland KE, Sheline YI, Weiss ES. Depression and coronary heart disease: a review for cardiologists. Clin Cardiol 1997; 20:196-200.

28. Yeragani VK, Tancer M, Seema KP, Josyula K, Desai N. Increased pulse-wave velocity in patients with anxiety: implications for autonomic dysfunction. J Psychosom Res 2006;61:25-31.

29. Whiteman MC, Deary IJ, Fowkes FG. Personality and social predictors of atherosclerotic progression: Edinburgh Artery Study. Psychosom Med 2000;62:703-714.

30. Chan TH, Ward S. Coping process theory: a tool to reduce stress and cardiovascular disease. AAOHN J 1993;41:499-503.

31. Wright TA, Sweeney D. Coping strategies and diastolic blood pressure. Psychol Rep 1989;65:443-449. 